\title{
CHARACTERIZATION OF EXTERNAL LOAD IN DIFFERENT TYPES OF EXERCISE IN PROFESSIONAL SOCCER
}

original paper

() Wroclaw University of Health and Sport Sciences

DOI: https://doi.org/10.5114/hm.2021.104190

\section{LILLIAN GONÇALVES ${ }^{1}$, MIGUEL CAMÕES ${ }^{1,2}$, RICARDO LIMA ${ }^{1,2}$, PEDRO BEZERRA $^{1,2}$, PANTELIS THEODOROS NIKOLAIDIS ${ }^{3}$, THOMAS ROSEMANN ${ }^{4}$, BEAT KNECHTLE ${ }^{5}$, FILIPE MANUEL CLEMENTE ${ }^{1,6}$}

\author{
${ }^{1}$ Escola Superior Desporto e Lazer, Instituto Politécnico de Viana do Castelo, Viana do Castelo, Portugal \\ ${ }^{2}$ Research Center in Sports Sciences, Health Sciences and Human Development, Vila Real, Portugal \\ ${ }^{3}$ Exercise Physiology Laboratory, Nikaia, Greece \\ ${ }^{4}$ Institute of Primary Care, University of Zurich, Zurich, Switzerland \\ ${ }^{5}$ Medbase St. Gallen Am Vadianplatz, St. Gallen, Switzerland \\ ${ }^{6}$ Instituto de Telecomunicações, Delegação da Covilhã, Covilhã, Portugal
}

\begin{abstract}
Purpose. To compare the duration of exercise and magnitude of external load per type of exercise (warm-up, small-sided games, position games - movements with/without ball but without the dynamics of a real game, simulations of real games - 11 vs. 11 , fitness exercises, large-sided games, technical drills) during different training days in professional soccer players and test the relationships between duration of exercise and the external load.

Methods. Overall, 22 professional soccer players $(25.1 \pm 2.9$ years, $181.9 \pm 6.3 \mathrm{~cm}, 73.1 \pm 6.3 \mathrm{~kg})$ were daily monitored by a microelectromechanical system. The training duration (minutes) and the external load measures of total distance covered and high-speed running distance were collected for each type of exercise.

Results. Repeated measures ANOVA revealed significant differences in training duration, total distance covered, and high-speed running distance between the exercises $(p<0.001)$. A greater total distance was covered in simulations of real games $(2321 \mathrm{~m} / \mathrm{session})$, as well as high-speed running $(84.6 \mathrm{~m} / \mathrm{session})$. The total distance covered in simulations of real games was greater by $77 \%$ than in small-sided games, while that in high-speed running in simulations of real games was greater by $153 \%$ than in large-sided games.
\end{abstract}

Conclusions. The study revealed that simulations of real games and position games were the exercises that occupied the most time in the sessions and that the simulations of real games were the greatest contributor to distance covered and distance covered at high-speed running.

Key words: association football, performance, training load, small-sided games, position games, sports training

\section{Introduction}

Training load monitoring is part of the training process allowing to daily characterize the impact of exercises on physical demands (external load) and biological responses (internal load) [1]. Commonly, both dimensions of load are analysed in an accumulated way (overall session), without consideration of the specific direction of load (type of exercises) [2]. However, a more detailed analysis (namely, considering the im- plications of each exercise) may help to identify the specific implications of particular types of exercise for different sports. Moreover, the use of technologies as microelectromechanical systems (MEMS) may turn easier the process of split and organize the external load for each exercise [3-6]. In the particular case of soccer, the monitoring can be extremely helpful, mainly because of the normal heterogeneity of load occurring in the same exercise.

Correspondence address: Filipe Manuel Clemente, Escola Superior Desporto e Lazer, Instituto Politécnico de Viana do Castelo, Rua Escola Industrial e Comercial de Nun’Álvares, 4900-347, Viana do Castelo, Portugal,

e-mail: filipe.clemente5@gmail.com

Received: June 11, 2020

Accepted for publication: December 17, 2020

Citation: Gonçalves L, Camões M, Lima R, Bezerra P, Nikolaidis PT, Rosemann T, Knechtle B, Clemente FM. Characterization of external load in different types of exercise in professional soccer. Hum Mov. 2022;23(1):89-95; doi: https://doi. org/10.5114/hm.2021.104190. 
As part of daily routines in soccer training, different exercises are proposed by coaches in order to achieve the session's goals. Owing to the variety of exercise types and, naturally, load direction, it is reasonable to assume that the typology of exercise will promote different loads and impacts on the soccer players. Thus, to properly manage the external load, coaches should be aware of the effects of different types of exercise and should aim to organize them in the most effective way on the most appropriate days [7, 8].

A simple organization of load direction would be to split the exercises into those more focused on physical dimensions and those more focused on technical/ tactical capacities. Among the technical/tactical types of exercise, the small-sided games (SSGs) are often used by coaches because of their capacity to simulate specific conditions of the match while imposing a considerable physiological stimulus on the players $[9,10]$. These games can promote different effects on players and for that reason are commonly organized in small (SSGs: 1 vs. 1 to 4 vs. 4), medium (MSGs: 5 vs. 5 to 7 vs. 7), and large (LSGs: 7 vs. 7 to 11 vs. 11) formats [11]. Other technical/tactical drills can include position games (PGs) (i.e., specific movements made by players with or without the ball, aiming to optimize the synchronization between teammates) or simulations of real games (RGs, i.e., games played in 11 vs. 11 formats during training sessions). Among the more focused exercises, there are running-based exercises, neuromuscular training, speed exercises, or coordination drills [12].

Each of the types of exercise naturally promotes a different stimulus in the players. Such stimulus (or load impact) should be described in order to understand how the organization of training (i.e., type of exercises, sequence, duration, intensity, volume) may explain the overall load on players. Despite the evidence regarding load variations during a week and the acute effects of specific games [13, 14], there is a lack of data about the load direction and the impact of different types of exercise on players [15]. Additionally, considering that the duration of exercise can be related to the volume of load, it is also important to characterize how much time is dedicated to each group of exercise. Such information could reflect how coaches manage exercises and the true impact of such exercises in terms of the final external load experienced by players (e.g., distance- and accelerometerybased measures). Finally, a consideration of how the load is imposed within a week is also interesting, namely to identify the within-week changes and fluctuations of the load distribution and exercises across a week (taking into account the proximity to the next match) [16].

In this context, the objective of the present study was to compare the duration of exercise and magnitude of external load per type of exercise during different training days in professional soccer players. Additionally, relationships between the duration of exercise and external load were tested in order to identify if these could be related. It was hypothesized that game-based drills (e.g., PGs, SSGs, RGs) would have greater duration and volume of external load than strength and conditioning exercises and analytical drills [15]. Moreover, a significant correlation was hypothesized between the duration of exercise and the external load performed.

\section{Material and methods}

\section{Participants}

Overall, 22 professional soccer players $(25.1 \pm 2.9$ years, $181.9 \pm 6.3 \mathrm{~cm}, 73.1 \pm 6.3 \mathrm{~kg}$ ) from a Portuguese professional team competing in the first league voluntarily participated in this study. The study followed the ethical recommendations for research in humans as suggested by Harris et al. [17]. The following inclusion criteria were applied: (i) all the players were present at a minimum of $90 \%$ of the training sessions that occurred during the period of analysis (5 months); (ii) to be included in the data treatment of any given week, the player had to be present at all training sessions of that week; (iii) only the training sessions in which all the players participated in the same exercises were included (the training sessions for players who did not play in the match were excluded from the analysis).

\section{Design and experimental procedures}

This study followed an observational and descriptive design. The data were collected for training sessions that occurred between September 2018 and January 2019 (early and mid-season), by using a MEMS (V4 tracker, JOHAN Sports, Noordwijk, the Netherlands) that consisted of a GPS sensor $(10 \mathrm{~Hz}$, including EGNOS correction), an accelerometer, a gyroscope, and a magnetometer $(100 \mathrm{~Hz}, 3$ axes, $\pm 16 \mathrm{~g})$. The specific MEMS applied in this study was previously tested for validity and reliability; the difference between GPS and $200-\mathrm{m}$ distance was $-0.13 \pm 3.94 \mathrm{~m}$ to $2.13 \pm$ $2.64 \mathrm{~m}$ and inter-unit coefficient of variation ranged from $2.08 \%$ to $3.92 \%$ [18]. 
Table 1. Number of times each type of exercise occurred during the observation period

\begin{tabular}{lccccc}
\hline Exercise & September $(n)$ & October $(n)$ & November $(n)$ & December $(n)$ & January $(n)$ \\
\hline Warm-up & 17 & 16 & 20 & 13 & 12 \\
Small-sided games & 9 & 16 & 7 & 6 & 3 \\
Position games & 3 & 5 & 6 & 4 & 7 \\
Simulations of real game & 10 & 9 & 3 & 5 & 3 \\
Fitness exercises & 8 & 4 & 10 & 7 & 9 \\
Large-sided games & 10 & 8 & 7 & 10 & 11 \\
Technical drills & 6 & 13 & 8 & 7 \\
\hline
\end{tabular}

The training sessions of the week were defined as MD-1 (match day -1: one day before a match), MD-2 (match day -2: two days before a match), MD-3 (match day -3: three days before a match), MD-4 (match day -4: four days before a match), and MD-5 [match day -5 : five days before a match). During the observation period, 10 sessions occurred at MD-1, 17 at MD-2, 17 at MD-3, 14 at MD-4, and 7 at MD-5.

All the exercises were characterized and described by the staff, and a specific type of exercise from the following list was added: (i) warm-up (WU: exercises aimed to prepare the players for the fundamental part of the session; examples: jogging, mobility or dynamic stretching or post-activation potentiation); (ii) SSGs (sided-games that varied between the 1 vs. 1 and the 5 vs. 5 formats); (iii) PGs (exercises that promoted the collective organization of players without a defending-attacking free dynamics); (iv) RGs (11 vs. 11 free games disputed between teammates and following the regular rules); (v) fitness exercises (FEs: analytical exercises, with or without a ball, with the main focus of developing a specific physical quality, such as velocity, coordination, sprinting, or high-intensity running); (vi) LSGs (sided-games larger than the 5 vs. 5 format); and (vii) technical drills (TDs: analytical and drillbased exercises mainly focused on developing technical aspects, such as passing and shooting). The number of times each type of exercise was performed during the observation period can be found in Table 1 .

\section{External load measures}

The same MEMS was used by each player during the study period to reduce the inter-variability error of the devices. The participants wore the GPS in a pocket within a skin-tight vest on the back (between the scapulae). The data collected during the training sessions were imported and treated in the JOHAN Sports web application, and after each training session, the data were divided depending on the type of exercise. The following variables were collected, with the thresholds defined by the devices: (i) total distance covered (the total number of meters covered by a player during the exercise); and (ii) high-speed running distance (the distance covered at a speed of $19.8-24.9 \mathrm{~km} / \mathrm{h}$ ). The absolute distance covered $(\mathrm{m})$ in each measure per type of exercise was recorded. The total distance covered was selected because it represents a measure of overall distance covered in the session/exercise, while highspeed running distance was chosen since this specific velocity threshold represents the point of running intensification (namely, the entrance in the anaerobic speed reserve for some players) [19].

\section{Statistical procedures}

The data were firstly tested for normality and homogeneity by using the Kolmogorov-Smirnov test and Levene's test for $p>0.05$. Descriptive statistics were calculated and presented in the results in the form of mean and standard deviation. The comparisons of the volume of training (duration in minutes), load measures (total distance covered and high-speed running distance) and day of training session were tested with repeated measures ANOVA followed by the Bonferroni post-hoc test after confirmation of normality and homogeneity of the sample. Correlations between the total time and distance-based measures were established by using Pearson's correlation coefficient, with the following interpretation of magnitudes: [0.0;0.1], trivial; [0.1;0.3], small; [0.3;0.5], moderate; [0.5;0.7], large; $[0.7 ; 0.9]$, very large; and $[0.9 ; 1.0]$, nearly perfect. The inferential tests were performed with the SPSS software (version 24.0, IBM, USA). Statistical significance of the results was accepted at $p<0.05$. The effect size of pairwise comparisons was tested with Cohen's $d$, with the following thresholds [20]: [0;0.2], trivial; [0.2;0.6], small; [0.6;1.2], moderate; [1.2;2.0], large; and $>2.0$, very large.

\section{Ethical approval}

The research related to human use has complied with all the relevant national regulations and institutional policies, has followed the tenets of the Declara- 


\section{HUMAN MOVEMENT}

L. Gonçalves et al., External load of different soccer tasks

tion of Helsinki, and has been approved by the School of Sport and Leisure (Melgaço, Portugal) scientific council.

\section{Informed consent}

Informed consent has been obtained from all individuals included in this study.

\section{Results}

Repeated measures ANOVA revealed significant differences in training duration between the types of exercise $(p<0.001)$. The descriptive statistics can be found in Table 2. RGs were significantly longer than $\mathrm{WU}(p<0.001 ; d=2.240$, very large effect), SSGs $(p<$ $0.001 ; d=1.201$, large effect), PGs $(p<0.001 ; d=1.190$, moderate effect), FEs $(p<0.001 ; d=2.018$, very large effect), LSGs ( $p<0.001 ; d=1.402$, large effect), and TDs ( $p<0.001 ; d=1.710$, large effect) at MD-2.

At MD-3, it was found that, among others, PGs were the exercises with significantly longer duration comparing with WU $(p<0.001 ; d=11.108$, very large effect), SSGs $(p<0.001 ; d=12.000$, very large effect), RGs ( $p<0.001 ; d=5.036$, very large effect), LSGs $(p<$ $0.001 ; d=8.065$, very large effect), and TDs $(p<0.001$; $d=11.794$, very large effect).

Considering MD-4, it was observed that RGs occupied more time in the session in comparison with WU $(p<0.001 ; d=3.729$, very large effect), SSGs $(p<0.001$; $d=0.839$, moderate effect), PGs $(p<0.001 ; d=2.035$, very large effect), FEs $(p<0.001 ; d=2.966$, very large effect), LSGs ( $p<0.001 ; d=1.795$, large effect), and TDs ( $p<0.001 ; d=3.465$, very large effect).

Repeated measures ANOVA revealed significant differences in total distance between the types of exercise $(p<0.001)$. The descriptive statistics can be found in Table 3. Overall, it was observed that RGs contributed to the total distance significantly more than WU $(p<0.001 ; d=1.686$, large effect), SSGs $(p<0.001$; $d=1.009$, moderate effect), PGs $(p<0.001 ; d=0.999$, moderate effect), FEs ( $p<0.001 ; d=1.505$, large effect), LSGs ( $p<0.001 ; d=1.151$, moderate effect), and TDs $(p<0.001 ; d=1.612$, large effect).

Repeated measures ANOVA revealed significant differences in high-speed running distance between the types of exercise $(p<0.001)$. The descriptive statistics can be found in Table 4. Overall, pairwise comparisons demonstrated that RGs were a greater contributor to the high-speed running distance comparing with WU ( $p<0.001 ; d=1.395$, large effect), SSGs $(p<0.001 ; d=0.827$, moderate effect), PGs $(p<0.001$; $d=0.873$, moderate effect), FEs $(p<0.001 ; d=0.891$, moderate effect), LSGs $(p<0.001 ; d=0.818$, moderate effect), and TDs ( $p<0.001 ; d=1.265$, large effect).

Relationships between the duration (minutes) and the total distance and high-speed running distance

Table 2. Descriptive statistics (mean \pm standard deviation) of duration (minutes) per type of exercise

\begin{tabular}{lccccccc}
\hline Sessions & WU & SSGs & PGs & RGs & FEs & LSGs & TDs \\
\hline MD-1 & $12.0 \pm 5.0$ & - & $22.8 \pm 8.4$ & $18.7 \pm 5.0$ & $8.0 \pm 3.5$ & $11.7 \pm 3.5$ & $23.3 \pm 4.9$ \\
MD-2 & $13.0 \pm 5.0$ & $19.3 \pm 9.3$ & $18.7 \pm 5.3$ & $35.1 \pm 18.8$ & $6.9 \pm 2.4$ & $20.4 \pm 5.9$ & $15.3 \pm 4.9$ \\
MD-3 & $13.1 \pm 6.1$ & $17.3 \pm 5.3$ & $78.4 \pm 0.0$ & $25.8 \pm 10.9$ & $5.2 \pm 0.0$ & $18.8 \pm 7.5$ & $16.8 \pm 5.5$ \\
MD-4 & $13.0 \pm 5.2$ & $22.3 \pm 9.6$ & $18.8 \pm 0.0$ & $37.6 \pm 10.3$ & $10.2 \pm 7.0$ & $20.5 \pm 9.2$ & $11.8 \pm 3.7$ \\
MD-5 & $10.9 \pm 4.5$ & $19.0 \pm 5.7$ & $35.1 \pm 10.6$ & $23.1 \pm 11.4$ & $5.9 \pm 2.9$ & $15.8 \pm 1.2$ & $12.7 \pm 4.3$ \\
Overall & $12.7 \pm 5.4$ & $19.8 \pm 13.4$ & $25.0 \pm 14.4$ & $27.8 \pm 14.0$ & $7.5 \pm 3.9$ & $19.2 \pm 7.4$ & $16.2 \pm 6.1$ \\
\hline
\end{tabular}

WU - warm-up, SSGs - small-sided games, PGs - position games, RGs - simulations of real games,

FEs - fitness exercises, LSGs - large-sided games, TDs - technical drills

Table 3. Descriptive statistics (mean \pm standard deviation) of total distance (meters) per type of exercise

\begin{tabular}{|c|c|c|c|c|c|c|c|}
\hline Sessions & WU & SSGs & PGs & RGs & FEs & LSGs & TDs \\
\hline MD-1 & $559 \pm 249$ & - & $1071 \pm 411$ & $1336 \pm 365$ & $339 \pm 107$ & $428 \pm 204$ & $677 \pm 275$ \\
\hline MD-2 & $821 \pm 395$ & $1285 \pm 741$ & $896 \pm 205$ & $2746 \pm 1666$ & $299 \pm 140$ & $1260 \pm 597$ & $748 \pm 226$ \\
\hline MD-3 & $1077 \pm 516$ & $1316 \pm 560$ & $3901 \pm 432$ & $2385 \pm 1043$ & $731 \pm 44$ & $1005 \pm 709$ & $706 \pm 161$ \\
\hline MD-4 & $924 \pm 381$ & $1306 \pm 730$ & $1328 \pm 260$ & $3093 \pm 1726$ & $697 \pm 267$ & $1334 \pm 957$ & $645 \pm 132$ \\
\hline MD-5 & $943 \pm 529$ & $1412 \pm 378$ & $1972 \pm 337$ & $2036 \pm 1184$ & $576 \pm 114$ & $1096 \pm 225$ & $733 \pm 306$ \\
\hline Overall & $879 \pm 456$ & $1309 \pm 671$ & $1242 \pm 730$ & $2321 \pm 1373$ & $428 \pm 208$ & $1136 \pm 737$ & $710 \pm 231$ \\
\hline
\end{tabular}

WU - warm-up, SSGs - small-sided games, PGs - position games, RGs - simulations of real games,

FEs - fitness exercises, LSGs - large-sided games, TDs - technical drills 
Table 4. Descriptive statistics (mean \pm standard deviation) of high-speed running distance (meters) per type of exercise

\begin{tabular}{|c|c|c|c|c|c|c|c|}
\hline Sessions & WU & SSGs & PGs & RGs & FEs & LSGs & TDs \\
\hline MD-1 & $1.2 \pm 4.5$ & - & $22.5 \pm 25.9$ & $22.5 \pm 19.6$ & $2.3 \pm 6.0$ & $1.9 \pm 4.6$ & $0.2 \pm 0.6$ \\
\hline MD-2 & $2.9 \pm 7.2$ & $21.4 \pm 31.4$ & $16.6 \pm 21.2$ & $95.5 \pm 99.5$ & $10.3 \pm 11.8$ & $34.3 \pm 47.9$ & $6.6 \pm 9.3$ \\
\hline MD-3 & $2.8 \pm 7.4$ & $36.2 \pm 37.6$ & $61.3 \pm 42.6$ & $103.1 \pm 68.4$ & $0.0 \pm 0.0$ & $21.9 \pm 33.3$ & $5.2 \pm 9.4$ \\
\hline MD-4 & $21.8 \pm 9.8$ & $36.5 \pm 39.9$ & $26.5 \pm 25.4$ & $115.0 \pm 119.7$ & $91.7 \pm 68.6$ & $53.2 \pm 52.8$ & $8.7 \pm 12.6$ \\
\hline MD-5 & $29.1 \pm 8.7$ & $27.0 \pm 30.2$ & $54.2 \pm 49.6$ & $74.7 \pm 83.2$ & $37.7 \pm 36.6$ & $55.3 \pm 94.2$ & $13.1 \pm 24.9$ \\
\hline Overall & $8.6 \pm 37.4$ & $31.5 \pm 36.9$ & $25.7 \pm 31.2$ & $84.6 \pm 87.4$ & $20.1 \pm 40.0$ & $33.4 \pm 48.7$ & $6.5 \pm 13.5$ \\
\hline
\end{tabular}

WU - warm-up, SSGs - small-sided games, PGs - position games, RGs - simulations of real games,

FEs - fitness exercises, LSGs - large-sided games, TDs - technical drills

(a) 1

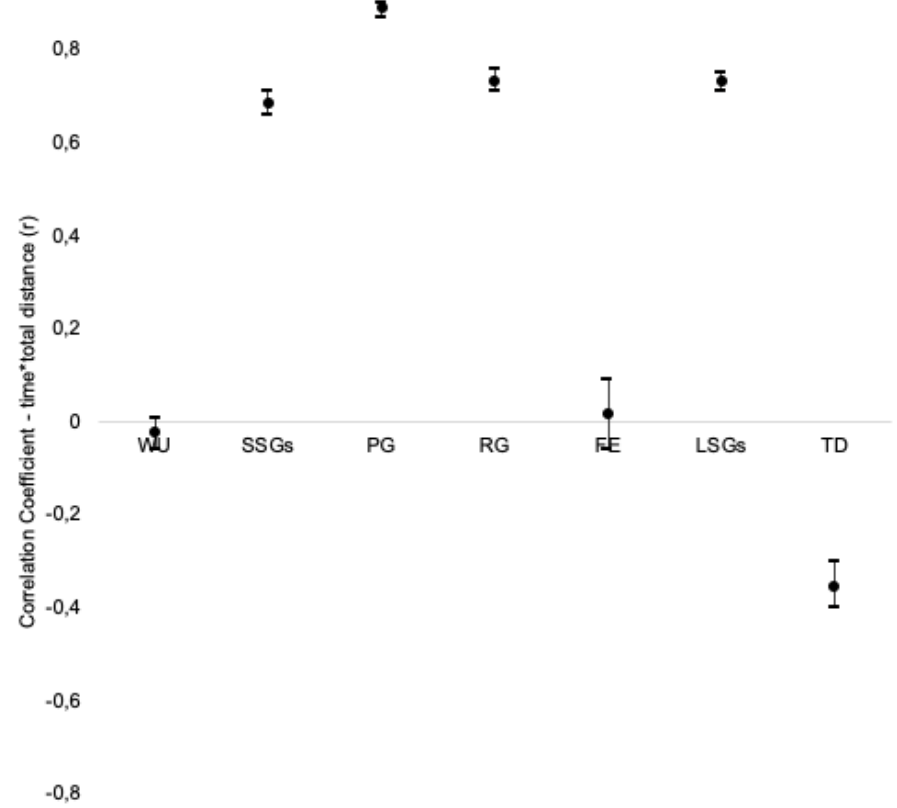

(b) 1

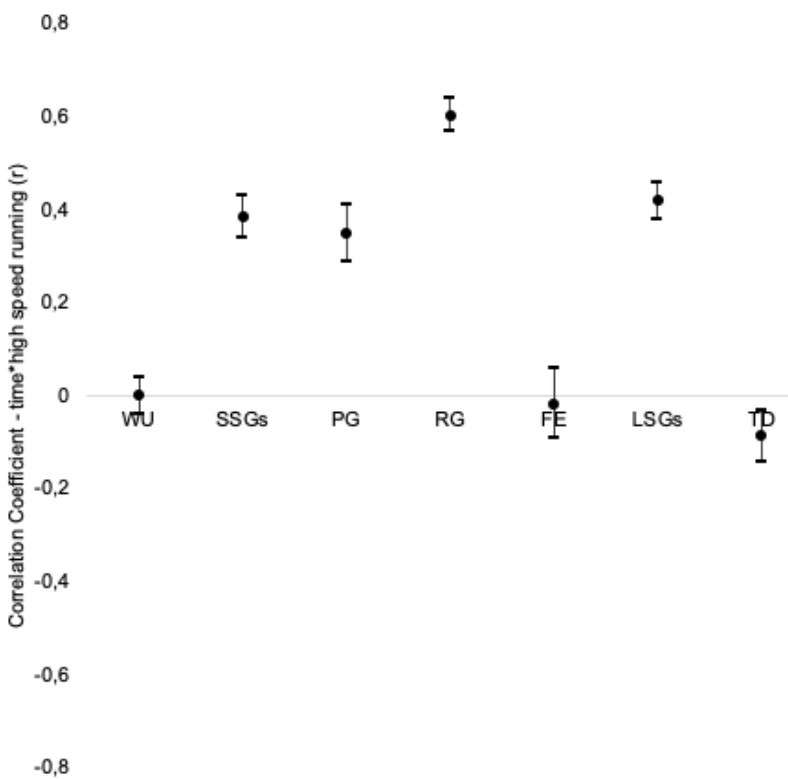

$-1$

WU - warm-up, SSGs - small-sided games, PG - position games, RG - simulations of real games, FE - fitness exercises, LSGs - large-sided games, TD - technical drills

Figure 1. Correlation coefficients ( $r$ ) between duration and (a) total distance and (b) high-speed running distance, by type of exercise

were tested. The results of such correlations can be found in Figure 1. Very large correlations (greater than $r=0.7)$ were observed between duration and total distance for PGs, RGs, and LSGs. As for correlations between high-speed running distance and duration, only large correlations (greater than $r=0.5$ ) were revealed for RGs.

\section{Discussion}

The purpose of this study was to characterize the duration of exercise and the volume of external load per type of exercise during different training days in professional soccer players. The main findings re- vealed that duration, total distance, and high-speed running distance were significantly different between the types of exercise. Specifically, RGs were the exercise with the highest duration in the sessions, as well as imposed greater values of total distance and high-speed running distance.

Considering the specific measure of exercise duration, RGs and PGs had a greater amount of time during the sessions, followed by SSGs and LSGs. Such types of exercise are more representative of the official matches and possibly this could explain the great amount of time dedicated to them within the session. RGs also exhibited greater volumes of total distance and high-speed running distance. This could be ex- 
plained by the greater amount of time spent on these exercises, as well by their capacity to represent the physical demands of official games, e.g., the distances covered at different speed thresholds.

Interestingly, despite the great amount of time spent on SSGs and LSGs, there were no significant differences with PGs and the comparison with FEs resulted in small magnitudes. In fact, SSGs and LSGs seem not to elicit great amounts of intense running by the fact of not involving enough space to produce such actions $[21,22]$. This could be highly considered mainly because in a smaller amount of time, analytical methods (e.g., running-based high-intensity interval training) may provide a more convenient stimulus for specific physical qualities [23]. Another option may be a combination of SSGs or LSGs and running-based activities to ensure achieving a necessary mechanical dose of training in a smaller period of time [24].

Following such an idea, the relationships between the duration of exercise and external load demands also suggested that SSGs and LSGs had moderate correlations with high-speed running distance. On the other hand, these drills presented large correlations with total distance, which is in line with studies that suggest a great amount of distance accumulated in these formats of play [21, 25]. In turn, RGs exhibited a greater magnitude of correlations between exercise duration and total distance and high-speed running distance. Curiously, the correlations between load and exercise duration were trivial for WU, FEs, and TDs. In the specific case of FEs, this can be justified by the fact that this category involved many exercises related to physical qualities (e.g., neuromuscular, training circuit) in which there was not necessarily a great amount of speed or even distance.

This study had some limitations. The sample size does not allow us to generalize the evidence since only one team was analysed. Naturally, at the professional level, it is difficult to collect more than one team; for that reason, more studies are necessary to properly characterize the load distribution depending on the type of exercise. Moreover, sub-categories may be investigated in future studies, combining drill-based exercises. Finally, internal load should also be monitored in further research, as well as more external load measures, such as sprinting and the number of accelerations and decelerations. Additionally, information about exercises performed without horizontal displacement should be monitored by using inertial measurement units that provide data on general load based on triaxial acceleration. Despite the limitations, this study is, to the best of our knowledge, the first one characterizing load distribution within training sessions and as such may be used as a starting point for new analyses and interpretations. Possibly, general workload indices may be refined with a weight analysis based on load distribution. Besides, using this information, coaches may consider implementing exercises in a more appropriate way in the planning process, namely considering the general effect on professional players.

\section{Conclusions}

This study revealed significant differences in duration and external loads per type of exercise, thus suggesting a differentiation in load distribution. Comparisons between the types of exercise demonstrated that RGs and PGs were the exercises that occupied the most time in the sessions and that RGs imposed a greater amount of external load. In practical applications, general workload indices may be refined with a weight analysis based on load distribution.

\section{Acknowledgements}

We would like to thank Nuno Pinto for helping in data collection and JOHAN Sports for providing the GPS system for data collection. This study made part of one curricular unit of Master in Sports Training at Escola Superior de Desporto e Lazer, Instituto Politécnico de Viana do Castelo, Portugal.

\section{Funding}

This work was funded by Fundação para a Ciência e a Tecnologia of the Portuguese Ministry of Science, Technology, and Higher Education (FCT/MCTES) through national funds and, when applicable, co-funded by European Union funds under the project UIDB/ EEA/50008/2020.

\section{Disclosure statement}

No author has any financial interest or received any financial benefit from this research.

\section{Conflict of interest}

The authors state no conflict of interest.

\section{References}

1. Impellizzeri FM, Marcora SM, Coutts AJ. Internal and external training load: 15 years on. Int J Sports Physiol Perform. 2019;14(2):270-273; doi: 10.1123/ ijspp.2018-0935.

2. Burgess DJ. The research doesn't always apply: practical solutions to evidence-based training-load monitoring in elite team sports. Int J Sports Physiol Perform. 
2017;12(Suppl. 2):S2136-S2141; doi: 10.1123/ijspp. 2016-0608.

3. Gaudino P, Iaia FM, Alberti G, Strudwick AJ, Atkinson G, Gregson W. Monitoring training in elite soccer players: systematic bias between running speed and metabolic power data. Int J Sports Med. 2013;34(11): 963-968; doi: 10.1055/s-0033-1337943.

4. Portas MD, Harley JA, Barnes CA, Rush CJ. The validity and reliability of $1-\mathrm{Hz}$ and $5-\mathrm{Hz}$ global positioning systems for linear, multidirectional, and soccer-specific activities. Int J Sports Physiol Perform. 2010;5(4):448458; doi: 10.1123/ijspp.5.4.448.

5. Randers MB, Mujika I, Hewitt A, Santisteban J, Bischoff R, Solano R, et al. Application of four different football match analysis systems: a comparative study. J Sports Sci. 2010;28(2):171-182; doi: 10.1080/0264 0410903428525.

6. Waldron M, Worsfold P, Twist C, Lamb K. Concurrent validity and test-retest reliability of a global positioning system (GPS) and timing gates to assess sprint performance variables. J Sports Sci. 2011;29(15):1613-1619; doi: 10.1080/02640414.2011.608703.

7. Impellizzeri FM, Rampinini E, Marcora SM. Physiological assessment of aerobic training in soccer. J Sports Sci. 2005;23(6):583-592; doi: 10.1080/02640 410400021278.

8. Scott TJ, Black CR, Quinn J, Coutts AJ. Validity and reliability of the session-RPE method for quantifying training in Australian football. J Strength Cond Res. 2013;27(1):270-276; doi: 10.1519/JSC.0b013e318254 $1 \mathrm{~d} 2 \mathrm{e}$.

9. Sarmento H, Clemente FM, Harper LD, da Costa IT, Owen A, Figueiredo AJ. Small sided games in soccer - a systematic review. Int J Perform Anal Sport. 2018;18(5): 693-749; doi: 10.1080/24748668.2018.1517288.

10. Clemente FM, Sarmento H. The effects of small-sided soccer games on technical actions and skills: a systematic review. Hum Mov. 2020;21(3):100-119; doi: 10.5114/ hm.2020.93014.

11. Owen AL, Wong DP, Paul D, Dellal A. Physical and technical comparisons between various-sided games within professional soccer. Int J Sports Med. 2014; 35(4):286-292; doi: 10.1055/s-0033-1351333.

12. Reilly T. The science of training - soccer. Abingdon: Routledge; 2007.

13. Clemente FM, Mendes B, Nikolaidis PT, Calvete F, Carriço S, Owen AL. Internal training load and its longitudinal relationship with seasonal player wellness in elite professional soccer. Physiol Behav. 2017;179:262267; doi: 10.1016/j.physbeh.2017.06.021.

14. Kelly DM, Strudwick AJ, Atkinson G, Drust B, Gregson W. Quantification of training and match-load distribution across a season in elite English Premier League soccer players. Sci Med Footb. 2020;4(1):59-67; doi: 10.1080/24733938.2019.1651934.

15. Jeong T-S, Reilly T, Morton J, Bae S-W, Drust B. Quantification of the physiological loading of one week of "pre-season" and one week of "in-season" training in professional soccer players. J Sports Sci. 2011;29(11): 1161-1166; doi: 10.1080/02640414.2011.583671.

16. Clemente FM, Oliveira H, Vaz T, Carriço S, Calvete F, Mendes B. Variations of perceived load and well-being between normal and congested weeks in elite case study handball team. Res Sports Med. 2019;27(3):412-423; doi: 10.1080/15438627.2018.1530998.

17. Harriss DJ, MacSween A, Atkinson G. Ethical standards in sport and exercise science research: 2020 update. Int J Sports Med. 2019;40(13):813-817; doi: 10.1055/a-1015-3123.

18. Nikolaidis PT, Clemente FM, van der Linden CMI, Rosemann T, Knechtle B. Validity and reliability of $10-\mathrm{Hz}$ global positioning system to assess in-line movement and change of direction. Front Physiol. 2018;9:228; doi: 10.3389/fphys.2018.00228.

19. Buchheit M, Laursen PB. High-intensity interval training, solutions to the programming puzzle: Part I: cardiopulmonary emphasis. Sports Med. 2013;43(5):313338; doi: 10.1007/s40279-013-0029-x.

20. Batterham AM, Hopkins WG. Making meaningful inferences about magnitudes. Int J Sports Physiol Perform. 2006;1(1):50-57; doi: 10.1123/ijspp.1.1.50.

21. Clemente FM, Sarmento H, Rabbani A, Van Der Linden CMI (Niels), Kargarfard M, Costa IT. Variations of external load variables between medium- and largesided soccer games in professional players. Res Sports Med. 2019;27(1):50-59; doi: 10.1080/15438627.2018. 1511560.

22. Clemente FM. The threats of small-sided soccer games: a discussion about their differences with the match external load demands and their variability levels. Strength Cond J. 2020;42(3):100-105; doi: 10.1519/SSC.0000 000000000526.

23. Buchheit M, Laursen PB. High-intensity interval training, solutions to the programming puzzle. Part II: anaerobic energy, neuromuscular load and practical applications. Sports Med. 2013;43(10):927-954; doi: 10.1007/s40279-013-0066-5.

24. Rabbani A, Clemente FM, Kargarfard M, Jahangiri S. Combined small-sided game and high-intensity interval training in soccer players: the effect of exercise order. J Hum Kinet. 2019;69(1):249-257; doi: 10.2478/hukin2018-0092.

25. Dalen T, Sandmæl S, Stevens TGA, Hjelde GH, Kjøsnes TN, Wisløff U. Differences in acceleration and highintensity activities between small-sided games and peak periods of official matches in elite soccer players. J Strength Cond Res. 2021;35(7):2018-2024; doi: 10.1519/JSC.0000000000003081. 